TRANSACTIONS OF THE

AMERICAN MATHEMATICAL SOCIETY

Volume 361, Number 10, October 2009, Pages 5601-5614

S 0002-9947(09)04870-3

Article electronically published on May 29, 2009

\title{
LIE MODELS FOR THE COMPONENTS OF SECTIONS OF A NILPOTENT FIBRATION
}

\author{
URTZI BUIJS, YVES FÉLIX, AND ANICETO MURILLO
}

\begin{abstract}
We give an explicit Lie model for any component of the space of free and pointed sections of a nilpotent fibration, and in particular, of the free and pointed mapping spaces. Among the applications presented, we obtain a Lie model of the exponential law and prove that, in many cases, the rank of the homotopy groups of the mapping space grows at the same rate as the rank of the homotopy groups of the target space.
\end{abstract}

\section{INTRODUCTION}

Let $F \rightarrow E \stackrel{p}{\rightarrow} X$ be a nilpotent fibration of nilpotent spaces in which the base is a finite complex. The purpose of this paper is to give a new way to compute the rational homotopy type of the $\operatorname{space} \operatorname{Sec}_{\sigma}(p)\left(\operatorname{resp} \cdot \operatorname{Sec}_{\sigma}^{*}(p)\right.$ ) of sections (resp. pointed sections) of $p$ that are homotopic to $\sigma$. In particular, when $p: X \times Y \rightarrow X$ is the projection and the section is given by a map $f: X \rightarrow Y$, these are the free and pointed mapping spaces $\operatorname{map}_{f}(X, Y)$ and $\operatorname{map}_{f}^{*}(X, Y)$.

Since the original work of Thom [15], much has been written on the subject, mostly from the contravariant Sullivan approach to rational homotopy. As a crucial contribution, a Sullivan model for $\operatorname{Sec}_{\sigma}(p)$ was first constructed by Haefliger [9] and rebuilt in a more functorial way by Brown and Szczarba in [2]. From those models, explicit descriptions of the rational homotopy groups of $\operatorname{map}_{f}(X, Y)$ have been obtained in [4] and [1]. For instance, if $\varphi: L_{X} \rightarrow L_{Y}$ is a Quillen model for $f$, Lupton and Smith prove that $\pi_{*} \operatorname{map}_{f}^{*}(X, Y) \otimes \mathbb{Q}$ is isomorphic to the homology of the complex of $\varphi$-derivations from $L_{X}$ into $L_{Y}$. However, from the Quillen setting based in differential Lie algebras, there are only partial descriptions. In this paper, we construct Lie models for the free and pointed sections of $p$, and in particular for mapping spaces, extending the work of Tanré and Scherer [13] on the constant component of the based mapping space.

Let $B$ be a finite dimensional CDGA model for $X$, let $L$ be a Lie model for the fiber $F$ and consider a model of $p$ of the form $B \rightarrow\left(B \otimes C^{*}(L), D\right)$, in which $C^{*}(L)=(\Lambda V, d)$ is the usual cochain algebra on $L$. Moreover (see end of $\S 1$ ) we

Received by the editors February 11, 2008.

2000 Mathematics Subject Classification. Primary 55P62, 54C35.

Key words and phrases. Space of sections, mapping space, Sullivan model, Quillen model, rational homotopy theory.

The first and third authors were partially supported by the Ministerio de Educación y Ciencia grant MTM2007-60016 and by the Junta de Andalucía grant FQM-213.

(C)2009 American Mathematical Society Reverts to public domain 28 years from publication 
may choose $\left(B \otimes C^{*}(L), D\right)$ to be a quotient of a cochain algebra on a Lie model of the total space and therefore its differential satisfies $D v=d v+\mathfrak{D} v+b$ where $b \in B$, $\mathfrak{D} v \subset B^{+} \otimes V$. We denote then by $B^{\#}$ the dual coalgebra with comultiplication $\Delta$, and by $\varphi: B \otimes C^{*}(L) \rightarrow B$ a model of the section $\sigma$. From $\varphi$, we deduce the following twisting function:

$$
\tilde{\varphi}: B^{\#} \stackrel{\varphi^{\#}}{\longrightarrow} C_{*}(L)=\Lambda s L \stackrel{\xi}{\longrightarrow} s L \stackrel{s^{-1}}{\longrightarrow} L
$$

in which $\xi: \Lambda s L \rightarrow s L$ is the projection on the indecomposables. We then make $\operatorname{Hom}\left(B^{\#}, L\right)$ a differential graded Lie algebra by defining the differential and the bracket as

$$
\begin{aligned}
{[f, g] } & =[] \circ(f \otimes g) \circ \Delta, \\
D(f) & =d \circ f+(-1)^{|f|} f \circ d-[\tilde{\varphi}, f]-\mathfrak{D}^{\#} \circ(1 \otimes f) \circ \Delta .
\end{aligned}
$$

Our main result then states (see Theorem 10 for the precise statement and the corresponding version for the pointed space of sections):

Theorem. The DGL $\operatorname{Hom}\left(B^{\#}, L\right)$ is a Lie model for the space $\operatorname{Sec}_{\sigma}(p)$.

Due to the naturality of this construction, it gives directly a description of the evaluation fibration $\operatorname{Sec}_{\sigma}^{*}(p) \rightarrow \operatorname{Sec}_{\sigma}(p) \rightarrow F$ (Theorem 14), and an exponential law exp: $\operatorname{map}(X \times Y, Z) \rightarrow \operatorname{map}\left(X, Z^{Y}\right)$ corresponding to the topological one (Theorem 17).

Moreover, being within the Quillen covariant approach to rational homotopy, these new models enable us to extract information deeply hidden in the Haefliger model, and explicit computations concerning the homotopy groups of the space of sections can be made (see Proposition 18 below). In particular we are able to show that, for coformal spaces $Y$ of finite Lusternik-Schnirelmann category, the growth rate of the rank of $\pi_{*} \operatorname{map}_{f}(X, Y)$ is, roughly speaking, the same as that of $\pi_{*}(Y)$ (see Theorem 20 for the precise statement):

Theorem. (i) If the image of the twisting cochain is contained in an ideal I with $\log$ index $I<\log$ index $L$, then

$$
\log \operatorname{index} \pi_{*}\left(\operatorname{map}_{f}(X, Y)\right) \otimes \mathbb{Q}=\log \operatorname{index} \pi_{*}(Y) .
$$

(ii) If the Lie algebra $\pi_{*}(\Omega Y) \otimes \mathbb{Q}$ has an infinite number of generators, then $\pi_{*}\left(\operatorname{map}_{f}(X, Y)\right) \otimes \mathbb{Q}$ is also infinite dimensional.

In the next section we present the Sullivan model of $\operatorname{Sec}_{\sigma}(p)$, which better suits our purposes. The bridge between the topology and our model is given by another Lie model of the space of sections which is based on " $\varphi$-derivations". In the second section we describe our new model and extract some consequences. Finally, applications and explicit computations on the rational homotopy of mapping spaces are given in $\S 3$.

\section{Sullivan MOdels FOR SPACES OF SECTIONS AND $\varphi$-DERIVATIONS}

Our results heavily depend on known facts and techniques arising from rational homotopy theory. All of them can be found with all details in [7. It is however necessary to make precise what a model for a nonconnected space means as long as 
all the path components of the considered space are nilpotent. Notice that, under our assumptions, this is the case for $\operatorname{Sec}(p)$ and $\operatorname{Sec}^{*}(p)$ as [12, Thm.4.1] shows that $\operatorname{Sec}_{\sigma}(p)$ and $\operatorname{Sec}_{\sigma}^{*}(p)$ are nilpotent.

In [14] (see also [1]), Sullivan introduces a couple of adjoint functors,

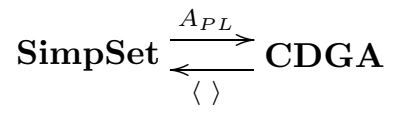

between the homotopy categories of commutative differential graded algebras and simplicial sets. As in [3, a model of a general space $X$ as above, not necessarily connected, is a $\mathbb{Z}$-graded free $\operatorname{CDGA}(\Lambda W, d)$ such that its simplicial realization $\langle(\Lambda W, d)\rangle$ has the same homotopy type as the Milnor simplicial approximation $S_{*}\left(X_{\mathbb{Q}}\right)$ of $X_{\mathbb{Q}}$. In the same way, a model for a map $f: X \rightarrow Y$ is a morphism of free CDGA's $(\Lambda U, d) \rightarrow(\Lambda W, d)$ such that its simplicial realization has the same homotopy type as the simplicial map $S_{*}\left(f_{\mathbb{Q}}\right)$. Note that, as the realization functor is not, in general, an equivalence, this definition does not preserve quasi-isomorphisms and thus, the model of a given nonconnected space does not provide its rational cohomology algebra (see Example 31). However, as explicitly described just after Theorem 1, it is possible to obtain a Sullivan model of any component $X_{i}$ of $X$ from a given model of $X$.

We start by introducing the Haefliger model 9 of the space of sections of a given fibration via the functorial description of Brown and Szczarba [2]. From now on, as stated in the introduction, $F \rightarrow E \stackrel{p}{\rightarrow} X$ shall denote a nilpotent fibration of nilpotent spaces in which the base $X$ is a finite complex. Moreover, when we deal with pointed mapping spaces or spaces of pointed sections, we assume that the fibre $F$ is simply connected.

Let $B$ be a finite dimensional CDGA (commutative differential graded algebra) model of the nilpotent finite complex $X$, and let $(B, d) \rightarrow(B \otimes \Lambda V, D) \rightarrow(\Lambda V, d)$ be a relative Sullivan algebra, or KS-extension, which models the nilpotent fibration $F \rightarrow E \stackrel{p}{\rightarrow} X$. We denote by $B^{\sharp}$ the differential coalgebra dual of $B$ with the grading $B^{\sharp-n}=B_{n}^{\sharp}=\operatorname{Hom}\left(B^{n}, \mathbb{Q}\right)$. Consider $\Lambda\left(B \otimes \Lambda V \otimes B^{\sharp}\right)$, the free commutative algebra generated by the $\mathbb{Z}$-graded vector space $(B \otimes \Lambda V) \otimes B^{\sharp}$ and endowed with the differential $d$ induced by $D$ and by the differential on $B^{\sharp}$. Let $J$ be the differential ideal generated by $1 \otimes 1-1$ and the elements of the form

$$
\begin{aligned}
& v_{1} v_{2} \otimes \beta-\sum_{j}(-1)^{\left|v_{2}\right|\left|\beta_{j}^{\prime}\right|}\left(v_{1} \otimes \beta_{j}^{\prime}\right)\left(v_{2} \otimes \beta_{j}^{\prime \prime}\right), \quad v_{1}, v_{2} \in V, \\
& b \otimes \alpha \otimes \beta-\sum_{j}(-1)^{\left|\beta_{j}^{\prime}\right|(|\alpha|+1)} \beta_{j}^{\prime}(b) \otimes \alpha \otimes \beta_{j}^{\prime \prime}, \quad b \in B, \alpha \in \Lambda V,
\end{aligned}
$$

where $\Delta \beta=\sum_{j} \beta_{j}^{\prime} \otimes \beta_{j}^{\prime \prime}$. The map induced by the inclusion $V \otimes B^{\sharp} \hookrightarrow B \otimes \Lambda V \otimes B^{\sharp}$,

$$
\rho: \Lambda\left(V \otimes B^{\sharp}\right) \stackrel{\cong}{\longrightarrow} \Lambda\left(B \otimes \Lambda V \otimes B^{\sharp}\right) / J
$$

is an isomorphism of graded algebras and thus $\tilde{d}=\rho^{-1} d \rho$ defines a differential in $\Lambda\left(V \otimes B^{\sharp}\right)$ for which:

Theorem $1([2,9]) \cdot\left(\Lambda\left(V \otimes B^{\sharp}\right), \tilde{d}\right)$ (resp. $\left.\left(\Lambda\left(V \otimes B_{+}^{\sharp}\right), \tilde{d}\right)\right)$ is a model of $\operatorname{Sec}(p)$ (resp. $\left.\operatorname{Sec}^{*}(p)\right)$. 
For the model of the components of $\operatorname{Sec}(p)$ and $/ o r \operatorname{Sec}^{*}(p)$ we follow the approach and notation of [3]:

For any free CDGA $(\Lambda W, d)$, in which $W$ is $\mathbb{Z}$-graded, and any algebra morphism $u: \Lambda W \longrightarrow \mathbb{Q}$ consider the differential ideal $K_{u}$ generated by $A_{1} \cup A_{2} \cup A_{3}$, where

$$
A_{1}=W^{<0}, A_{2}=d W^{0}, A_{3}=\left\{\alpha-u(\alpha): \alpha \in W^{0}\right\} .
$$

$(\Lambda W, d) / K_{u}$ is again a free CDGA of the form $\left(\Lambda\left(\bar{W}^{1} \oplus W^{\geq 2}\right), d_{u}\right)$ in which $\bar{W}^{1}$ is a complement in $W^{1}$ of $d\left(W^{0}\right)$ modulo identifications via $A_{1}$ and $A_{3}$ (see [3] for details). Note that $\bar{W}^{1}$ depends also on $u$. Moreover, if $(\Lambda W, d)$ is a model of a (nonconnected) space $X$ and $u$ corresponds to a 0 -simplex of $X$, as remarked in [3, 4.3], $\left(\Lambda\left(\bar{W}^{1} \oplus W^{\geq 2}\right), d_{u}\right)$ is a Sullivan model of the path component of $X$ containing the fixed 0-simplex.

We now apply this to the following: let $\sigma: X \rightarrow E$ be a section of $p$ which is modeled by a fixed retraction

$$
\phi:(B \otimes \Lambda V, D) \rightarrow(B, d) .
$$

This morphism restricts to an algebra map $\Lambda V \rightarrow B$ which determines an algebra morphism denoted also by

$$
\phi: \Lambda\left(V \otimes B^{\sharp}\right) \rightarrow \mathbb{Q} .
$$

Then, it turns out that:

Theorem $2([2,3])$. The projection

$$
\left(\Lambda\left(V \otimes B^{\sharp}\right), \tilde{d}\right) \longrightarrow\left(\Lambda\left(V \otimes B^{\sharp}\right), \tilde{d}\right) / K_{\phi} \cong\left(\Lambda\left({\overline{V \otimes B^{\sharp}}}^{1} \oplus\left(V \otimes B^{\sharp}\right)^{\geq 2}\right), d_{\phi}\right)
$$

is a model of the inclusion $\operatorname{Sec}_{\sigma}(p) \hookrightarrow \operatorname{Sec}(p)$. Moreover,

$$
\left(\Lambda\left(V \otimes B_{+}^{\sharp}\right), \tilde{d}\right) \longrightarrow\left(\Lambda\left(V \otimes B_{+}^{\sharp}\right), \tilde{d}\right) / K_{\phi} \cong\left(\Lambda\left({\overline{V \otimes B_{+}^{\sharp}}}^{1} \oplus\left(V \otimes B_{+}^{\sharp}\right)^{\geq 2}\right), d_{\phi}\right)
$$

is a model of the inclusion $\operatorname{Sec}_{\sigma}^{*}(p) \hookrightarrow \operatorname{Sec}^{*}(p)$.

In particular, $\left(\Lambda\left({\overline{V \otimes B^{\sharp}}}^{1} \oplus\left(V \otimes B^{\sharp}\right)^{\geq 2}\right), d_{\phi}\right)$ is a model of $\operatorname{Sec}_{\sigma}(p)$, which shall be denoted by $\left(\Lambda S, d_{\phi}\right)$. If $F$ is 1 -connected, then $\left(\Lambda(S / V), d_{\phi}\right)$ is a Sullivan model of $\operatorname{Sec}_{\sigma}^{*}(p)$.

Example 3. Consider the $\operatorname{space} \operatorname{map}\left(S^{n}, S^{n}\right), n$ odd, $n>1$, which is also $\operatorname{Sec}(p)$ for the trivial bundle $p: S^{n} \times S^{n} \rightarrow S^{n}$. A model of $S^{n}$ is $(\Lambda x, 0)$ with $|x|=n$, and a model of $\operatorname{map}\left(S^{n}, S^{n}\right)$ is $\left(\Lambda\left(x \otimes H_{*}\left(S^{n}\right)\right), 0\right)=(\Lambda(x, y), 0)$ with $|y|=0$. The 0 -simplices of the realization of this CDGA are given by

$$
\langle\Lambda(x, y)\rangle_{0}=\operatorname{Hom}(\mathbb{Q}[y], \mathbb{Q}) \cong \mathbb{Q} .
$$

The map $\mathbb{Q}[y] \rightarrow \mathbb{Q}, y \mapsto q$ corresponds to the component given by the maps of degree $q$ in $\operatorname{map}\left(S_{\mathbb{Q}}^{n}, S_{\mathbb{Q}}^{n}\right)$. The injection $\operatorname{map}_{q}\left(S_{\mathbb{Q}}^{n}, S_{\mathbb{Q}}^{n}\right) \hookrightarrow \operatorname{map}\left(S_{\mathbb{Q}}^{n}, S_{\mathbb{Q}}^{n}\right)$ is modeled by the projection $(\Lambda(x, y), 0) \rightarrow(\Lambda x, 0)$ that maps $y$ to $q$. Note that, although $(\Lambda(x, y), 0)$ is a model for the function space in the sense defined above, $H^{*}(\Lambda(x, y), 0)$ is isomorphic to neither $H^{*}\left(\operatorname{map}\left(S_{\mathbb{Q}}^{n}, S_{\mathbb{Q}}^{n}\right) ; \mathbb{Q}\right)$ nor $H^{*}\left(\operatorname{map}\left(S^{n}, S^{n}\right) ; \mathbb{Q}\right)$. In fact, as both $\operatorname{map}\left(S^{n}, S^{n}\right)$ and $\operatorname{map}\left(S_{\mathbb{Q}}^{n}, S_{\mathbb{Q}}^{n}\right)$ have infinitely many components, their rational homology in degree zero is infinite dimensional and, therefore, their rational cohomology in degree zero is not countable. 
Next, we introduce a vector space which gives, in general, the rational homotopy Lie algebra of $\operatorname{Sec}_{\sigma}(p)$ and, under some conditions, a Lie model for this space. Indeed, we consider the differential graded vector space $\left(\operatorname{Der}_{B}^{\phi}(B \otimes \Lambda V, B), \delta\right)$ of $\phi$-derivations over $B$, where $\operatorname{Der}_{B}^{\phi}(B \otimes \Lambda V, B)_{n}$ are the $B$-module maps of degree $n, \theta:(B \otimes \Lambda V)^{*} \rightarrow B^{*-n}$, for which $\theta(x y)=\theta(x) \phi(y)+(-1)^{n|x|} \phi(x) \theta(y), x, y \in V$. The differential is defined as usual, $\delta \theta=d \circ \theta+(-1)^{n+1} \theta \circ D$.

Observe that, as graded vector spaces, $\operatorname{Der}_{B}^{\phi}(B \otimes \Lambda V, B) \cong \operatorname{Hom}(V, B) \cong$ $\left(V \otimes B^{\sharp}\right)^{\sharp}$ via the canonical isomorphism $\Theta: \operatorname{Der}_{B}^{\phi}(B \otimes \Lambda V, B) \rightarrow\left(V \otimes B^{\sharp}\right)^{\sharp}$, $\Theta(\theta)(v \otimes \beta)=(-1)^{|\beta|(|v|+|\theta|)} \beta(\theta(v))$. From now on, we restrict to and focus on $\operatorname{Der}_{B}^{\phi}(B \otimes \Lambda V, B)$, the positive $\phi$-derivations over $B$,

$$
\mathcal{D}_{B}^{\phi}(B \otimes \Lambda V, B)_{i}=\left\{\begin{array}{cl}
\operatorname{Der}_{B}^{\phi}(B \otimes \Lambda V, B)_{i} & \text { for } i>1, \\
\operatorname{Der}_{B}^{\phi}(B \otimes \Lambda V, B)_{1} & \text { for } i=1,
\end{array}\right.
$$

in which $Z$ denotes the space of cycles. We shall also denote by $\delta$ the differential of this complex.

Given $\left(\Lambda\left({\overline{V \otimes B^{\sharp}}}^{1} \oplus\left(V \otimes B^{\sharp}\right)^{\geq 2}\right), d_{\phi}\right)=\left(\Lambda S, d_{\phi}\right)$, the model of $\operatorname{Sec}_{\sigma}(p)$ described above, for simplicity call it $d=d_{\phi}$ and, as for any Sullivan model, write $d=\sum_{i \geq 1} d_{i}$ in which $d_{i}(S) \subset \Lambda^{i} S$. Then, the same proof of [4, Thm. 5] produces:

Lemma 4. The following diagram commutes:

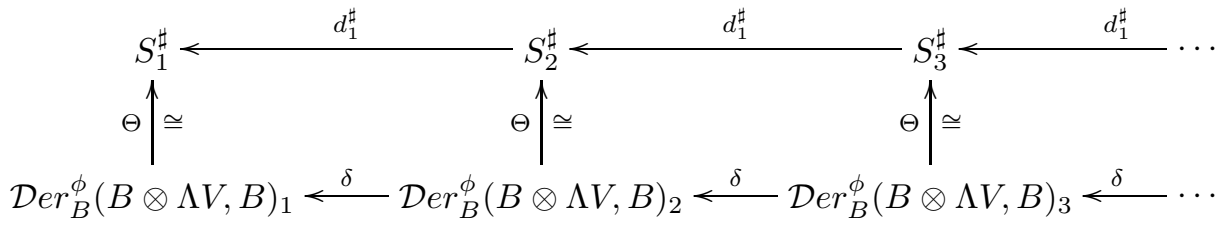

On the other hand, extending [4] we define the following.

Definition 5. Consider the linear map of degree 1,

$$
[,]: \mathcal{D}_{B}^{\phi}(B \otimes \Lambda V, B) \otimes \mathcal{D}_{B}^{\phi} r_{B}(B \otimes \Lambda V, B) \longrightarrow \mathcal{D}_{B}^{\phi}(B \otimes \Lambda V, B),
$$

defined by:

$$
[\varphi, \psi](v)=(-1)^{|\varphi|+|\psi|-1} \sum_{l} b_{l}\left(\sum_{i \neq j} \varepsilon \phi\left(v_{1} \ldots \hat{v}_{i} \ldots \hat{v}_{j} \ldots v_{k}\right) \varphi\left(v_{i}\right) \psi\left(v_{j}\right)\right),
$$

in which, for the differential $D$ in $B \otimes \Lambda V, D v=\sum_{l} b_{l} v_{1} \ldots v_{k}$ and $\varepsilon$ is the sign defined as follows: for each set of indices $\Gamma \subset\{1, \ldots, k\}$ write $\rho_{\Gamma}=\sum_{n \notin \Gamma}\left|v_{n}\right|$. For instance, $\rho_{\leq i, j}$ denotes $\left|v_{i+1}\right|+\cdots+\widehat{\left|v_{j}\right|}+\cdots+\left|v_{k}\right|$ if $i<j$ or $\left|v_{i+1}\right|+\cdots+\left|v_{k}\right|$ if $i \geq j$. Then,

$$
\varepsilon=(-1)^{\rho_{i, j}|\varphi|+\rho_{j}|\psi|+\rho_{\leq i, j}\left|v_{i}\right|+\rho_{\leq j}\left|v_{j}\right|} .
$$

This operation induces a bracket in homology with respect to which:

Lemma 6. $H_{*}\left(s^{-1} \mathcal{D} e r_{B}^{\phi}(B \otimes \Lambda V, B)\right)$ is a graded Lie algebra.

Proof. First, proceeding as in [4, Lemma 10], it is shown that, given derivations $\varphi, \psi \in \operatorname{Der}_{B}^{\phi}(B \otimes \Lambda V, B)$ and $v \otimes \beta \in S$,

$$
\Theta[\varphi, \psi](v \otimes \beta)=(-1)^{|\varphi|+|\psi|-1}\left\langle\Theta \varphi, \Theta \psi ; d_{2}(v \otimes \beta)\right\rangle .
$$


Here, $\langle, ;\rangle: S^{\sharp} \times S^{\sharp} \times \Lambda^{2} S \rightarrow \mathbb{Q}$ is the usual pairing. As $d_{2}$ induces a Lie algebra structure on $s^{-1} H^{*}\left(S, d_{1}\right)$, the lemma follows.

As an immediate consequence, Theorems 1 and 3 of [4] can be extended to:

Theorem 7. As Lie algebras, for $n \geq 2$,

$$
\begin{aligned}
& \pi_{n} \Omega \operatorname{Sec}_{\sigma}(p)_{\mathbb{Q}} \cong H_{n}\left(s^{-1} \operatorname{Der}_{B}^{\phi}(B \otimes \Lambda V, B), \delta\right), \\
& \pi_{n} \Omega \operatorname{Sec}_{\sigma}^{*}(p)_{\mathbb{Q}} \cong H_{n}\left(s^{-1} \mathcal{D e r}_{B}^{\phi}\left(B \otimes \Lambda V, B^{+}\right), \delta\right),
\end{aligned}
$$

while for $n=1$,

$$
\begin{aligned}
& \Gamma_{\mathbb{Q}}\left(\pi_{1} \operatorname{Sec}_{\sigma}(p)\right) \cong H_{1}\left(s^{-1} \mathcal{D e r}_{B}^{\phi}(B \otimes \Lambda V, B), \delta\right), \\
& \Gamma_{\mathbb{Q}}\left(\pi_{1} \operatorname{Sec}_{\sigma}^{*}(p)\right) \cong H_{1}\left(s^{-1} \mathcal{D e r}_{B}^{\phi}\left(B \otimes \Lambda V, B^{+}\right), \delta\right) .
\end{aligned}
$$

Here, as for any nilpotent space $Z, \Gamma_{\mathbb{Q}}\left(\pi_{1} Z\right)$ denotes the rational vector space $\bigoplus_{i=1}^{m} \Gamma_{i} / \Gamma_{i+1} \otimes \mathbb{Q}$, where $\pi_{1} Z=\Gamma_{1} \supset \Gamma_{2} \supset \cdots \supset \Gamma_{m}=\{1\}$ is the lower central series of $\pi_{1} Z$. In particular, $\operatorname{dim} \Gamma_{\mathbb{Q}}\left(\pi_{1} Z\right)=\operatorname{rk} \pi_{1} Z$.

It is important to note that, at the chain level, the bracket above does not define, in general, a differential graded Lie algebra (DGL henceforth) structure on $s^{-1} \operatorname{Der}_{B}^{\phi}(B \otimes \Lambda V, B)$ or $s^{-1} \mathcal{D e r}_{B}^{\phi}\left(B \otimes \Lambda V, B^{+}\right)$. Nevertheless, the following holds.

Lemma 8. If $\left(\Lambda V, d=d_{1}+d_{2}\right)$ has only linear and quadratic differentials, then, given $\varphi, \psi, \gamma \in \operatorname{Der}_{B}^{\phi}(B \otimes \Lambda V, B)$, $[\varphi, \psi]=(-1)^{|\varphi||\psi|}[\psi, \varphi]$;

$\delta[\varphi, \psi]+[\delta \varphi, \psi]+(-1)^{|\varphi|}[\varphi, \delta \psi]=0$

$(-1)^{|\varphi|}[\varphi,[\psi, \gamma]]+(-1)^{|\gamma|(|\varphi|+|\psi|+1)}[\gamma,[\varphi, \psi]]+(-1)^{|\psi|+|\varphi|(|\psi|+|\gamma|)}[\psi,[\gamma, \varphi]]=0$.

Proof. The first assertion is direct. Via Lemma 4 and formula (1) above, it is a straightforward (but tedious) calculation to show that the second and third assertions are equivalent to $d_{1} d_{2}+d_{2} d_{1}=0$ and $d_{2}^{2}=0$ respectively.

Now we recall the definition of the chain and cochain functors defined in the category of differential graded Lie algebras.

On the one hand, the Cartan-Eilenberg-Chevalley construction on a given DGL $\left(L, d_{L}\right)$ is the differential graded cocommutative coalgebra $C_{*}\left(L, d_{L}\right)=(\Lambda s L, d=$ $\left.d_{1}+d_{2}\right)$ in which, as usual, $(s L)_{k}=L_{k-1}$ and

$$
\begin{gathered}
d_{1}\left(s x_{1} \wedge \cdots \wedge s x_{k}\right)=-\sum_{i=1}^{k}(-1)^{n_{i}} s x_{1} \wedge \cdots \wedge s d_{L} x_{i} \wedge \cdots \wedge s x_{k}, \\
d_{2}\left(s x_{1} \wedge \cdots \wedge s x_{k}\right)=\sum_{1 \leq i<j \leq k}(-1)^{n_{i j}} s\left[x_{i}, x_{j}\right] \wedge s x_{1} \cdots s \hat{x_{i}} \cdots s \hat{x_{j}} \cdots \wedge s x_{k} .
\end{gathered}
$$

Here, $n_{i}=\sum_{j<i}\left|s x_{j}\right|$, and

$$
s x_{1} \wedge \cdots \wedge s x_{k}=(-1)^{n_{i j}} s x_{i} \wedge s x_{j} \wedge s x_{1} \cdots s \hat{x}_{i} \cdots s \hat{x}_{j} \cdots \wedge s x_{k} .
$$

The dual of the construction above is the commutative differential graded algebra $C^{*}\left(L, d_{L}\right)=\operatorname{Hom}\left(C_{*}\left(L, d_{L}\right), \mathbb{Q}\right)$. If $L$ is of finite type, then $C^{*}\left(L, d_{L}\right)=(\Lambda V, d)$, where $V$ and $s L$ are dual graded vector spaces and $d=d_{1}+d_{2}$ in which: $\left\langle d_{1} v ; s x\right\rangle=$ $(-1)^{|v|}\left\langle v ; s d_{L} x\right\rangle$ and $\left\langle d_{2} v ; s x \wedge s y\right\rangle=(-1)^{|y|+1}\langle v ; s[x, y]\rangle$. 
From now on we shall write, for convenience, $C_{*}(L)$ and $C^{*}(L)$ instead of $C_{*}\left(L, d_{L}\right)$ and $C^{*}\left(L, d_{L}\right)$.

Also, recall that, for a nilpotent space $X$ of rational homology of finite type, a Lie model is a DGL of finite type $\left(L, d_{L}\right)$ together with a quasi-isomorphism $C^{*}\left(L, d_{L}\right) \stackrel{\simeq}{\longrightarrow} A_{P L}(X)$.

Then, if we choose a model of the fibre of $p$ of the form $(\Lambda V, d)=C^{*}(L)$, with $L$ a Lie model of $F$, we have:

Theorem 9. The space $s^{-1} \mathcal{D e r}_{B}^{\phi}(B \otimes \Lambda V, B)\left(\right.$ resp. $\left.s^{-1} \mathcal{D e r}_{B}^{\phi}\left(B \otimes \Lambda V, B^{+}\right)\right)$endowed with the usual differential $\delta s^{-1} \varphi=-s^{-1} \delta \varphi$ and bracket $\left[s^{-1} \varphi, s^{-1} \psi\right]=$ $(-1)^{|\varphi|} s^{-1}[\varphi, \psi]$ is a Lie model of $\operatorname{Sec}_{\sigma}(p)$ (resp. $\operatorname{Sec}_{\sigma}^{*}(p)$ ).

Proof. Lemma 8 shows that $s^{-1} \mathcal{D e r}_{B}^{\phi}(B \otimes \Lambda V, B)\left(\right.$ resp. $\left.s^{-1} \mathcal{D e r}_{B}^{\phi}\left(B \otimes \Lambda V, B^{+}\right)\right)$ is in fact a DGL. To prove that $s^{-1} \operatorname{Der}_{B}^{\phi}(B \otimes \Lambda V, B)$ is a Lie model of $\operatorname{Sec}_{\sigma}(p)$ we show that $C^{*}\left(s^{-1} \mathcal{D e r}_{B}^{\phi}(B \otimes \Lambda V, B)\right) \cong\left(\Lambda S, d_{\phi}\right)$ as CDGA's. On the one hand, as algebras,

$$
\begin{aligned}
C^{*}\left(s^{-1} \operatorname{Der}_{B}^{\phi}(B \otimes \Lambda V, B)\right) & =\operatorname{Hom}\left(C_{*}\left(s^{-1} \mathcal{D e r}_{B}^{\phi}(B \otimes \Lambda V, B)\right), \mathbb{Q}\right) \\
& =\operatorname{Hom}\left(\Lambda \mathcal{D e r}_{B}^{\phi}(B \otimes \Lambda V, B), \mathbb{Q}\right) \\
& \cong \Lambda\left(\overline{V \otimes B^{\sharp}}{ }^{1} \oplus\left(V \otimes B^{\sharp}\right)^{\geq 2}\right)=\Lambda S,
\end{aligned}
$$

where the last isomorphism is provided by the canonical isomorphism $\Theta$. Next, observe that, as the differential of $\Lambda V=C^{*}(L)$ has only linear and quadratic parts, so does the differential $d_{\phi}$ in $\Lambda S$. Finally, it is straightforward, using Lemma 4 and formula (1), to show that the linear and quadratic parts of the differential in $\left(\Lambda S, d_{\phi}\right)$ are carried to the linear and quadratic parts of the differential on $C^{*}\left(s^{-1} \operatorname{Der}(\Lambda V, B ; \phi)\right)$. The same arguments apply to show that $s^{-1} \operatorname{Der}_{B}^{\phi}\left(B \otimes \Lambda V, B^{+}\right)$is a Lie model of $\operatorname{Sec}_{\sigma}^{*}(p)$.

Observe that a model of the fibration $p, B \rightarrow(B \otimes \Lambda V, D) \rightarrow(\Lambda V, d)$ can be chosen so that $D v=d v+\mathfrak{D} v+b, b \in B, \mathfrak{D} v \in B^{+} \otimes V$. This is obtained by applying the cochain functor to a surjective Lie model of $p, L_{E} \rightarrow L_{X}$, to get a relative Sullivan algebra, or KS-extension, $C^{*}\left(L_{X}\right) \rightarrow C^{*}\left(L_{E}\right)=C^{*}\left(L_{X}\right) \otimes \Lambda V$. Now, a finite dimensional model for $X$ is obtained by taking $B$ to be a finite dimensional CDGA quotient of $C^{*}\left(L_{X}\right)$ by some acyclic ideal.

\section{Lie Models For the COMPONENTS OF $\operatorname{SeC}_{\sigma}(\mathbf{p})$}

For the fibration $F \rightarrow E \stackrel{p}{\rightarrow} X$, and with the notation of the last observation of the previous section, write $C^{*}(L)=(\Lambda V, d)$ with $V=(s L)^{\sharp}$ and $L$ a model of $F$. As before, let $\phi: B \otimes C^{*}(L) \longrightarrow B$ be a model of the section $\sigma: X \rightarrow E$ of $p$ which restricts to an algebra morphism (not commuting with differentials!) $\phi: C^{*}(L) \rightarrow B$. We shall denote by $\tilde{\phi}$ the linear map (of lower degree -1 )

$$
\tilde{\phi}: B^{\sharp} \stackrel{\phi^{\sharp}}{\rightarrow} C_{*}(L)=\Lambda s L \stackrel{\xi}{\rightarrow} s L \stackrel{s^{-1}}{\rightarrow} L
$$

in which the composite $\Lambda s L \stackrel{\xi}{\rightarrow} s L \stackrel{s^{-1}}{\rightarrow} L$ is the canonical twisting cochain from the Chevalley-Eilenberg coalgebra on $L$ to $L$. In general, $\tilde{\phi}$ is a twisting cochain only when $\phi$, and thus $\phi^{\sharp}$ commutes with differentials. For convenience, we shall use the same notation for the restriction to $\tilde{\phi}: B_{+}^{\sharp} \rightarrow L$. 
Next, in the graded vector space $\operatorname{Hom}\left(B^{\sharp}, L\right)$, we consider the usual bracket $[f, g]=[,] \circ f \otimes g \circ \Delta$, and the perturbed differential $D_{\phi}=D-a d_{\tilde{\phi}}-\mathcal{R}$ :

$$
D_{\phi} f=\partial_{L} f+(-1)^{|f|} f \delta-[\tilde{\phi}, f]-\mathcal{R}_{f},
$$

where $\mathcal{R}_{f}=s^{-1} \mathfrak{D}^{\sharp} \circ\left(1_{B^{\sharp}} \otimes s f\right) \circ \Delta$. Finally, discard the negative graded part by defining $\mathcal{H o m}\left(B^{\sharp}, L\right)$ :

$$
\mathcal{H o m}_{i}\left(B^{\sharp}, L\right)=\left\{\begin{array}{cc}
\operatorname{Hom}_{i}\left(B^{\sharp}, L\right) & \text { for } i>1, \\
Z\left(\operatorname{Hom}_{1}\left(B^{\sharp}, L\right)\right) & \text { for } i=1 .
\end{array}\right.
$$

The same process is carried out to define $\mathcal{H o m}\left(B_{+}^{\sharp}, L\right)$. Then, our main result reads:

Theorem 10. The DGL Hom $\left(B^{\sharp}, L\right)$ (resp. $\mathcal{H o m}\left(B_{+}^{\sharp}, L\right)$ ) endowed with $D_{\phi}$ and $[$,$] is a Lie model for \operatorname{Sec}_{\sigma}(p)\left(\right.$ resp. $\left.\operatorname{Sec}_{\sigma}^{*}(p)\right)$.

Remark 11. As the vector space $\operatorname{Hom}\left(B^{\sharp}, L\right)$ is canonically isomorphic to $B \otimes L$, another formulation of this result is the following: consider a basis $\left\{b_{i}\right\}$ of $B$, its dual basis $\left\{\beta_{i}\right\}$, and write $\tilde{\phi}\left(\beta_{i}\right)=\ell_{i}$. Then, in $B \otimes L$ consider the usual bracket $\left[a \otimes \ell, b \otimes \ell^{\prime}\right]=(-1)^{|b||\ell|} a b \otimes\left[\ell, \ell^{\prime}\right]$ and the perturbed differential

$$
\delta_{\phi}(a \otimes \ell)=d a \otimes \ell+(-1)^{|a|} a \otimes \delta \ell+\sum_{i}(-1)^{\left|b_{i}\right||\ell|} a b_{i} \otimes\left[\ell, \ell_{i}\right]+\mathcal{R}(a \otimes \ell),
$$

where $\mathcal{R}: B \otimes L \rightarrow B \otimes L$ corresponds to the operator $\mathcal{R}$ through the isomorphism above. Finally, restrict to the DGL $\mathcal{L}^{\phi}$ :

$$
\mathcal{L}_{i}^{\phi}=\left\{\begin{array}{cc}
(B \otimes L)_{i} & \text { for } i>1 \\
Z(B \otimes L)_{1} & \text { for } i=1 .
\end{array}\right.
$$

The same process, starting with $\operatorname{Hom}\left(B_{+}^{\sharp}, L\right)$, produces the DGL $\mathcal{L}_{*}^{\phi}$.

Thus, one immediately obtains:

Corollary 12. The DGL's $\mathcal{L}^{\phi}$ and $\mathcal{L}_{*}^{\phi}$ are Lie models of $\operatorname{Sec}_{\sigma}(p)$ and $\operatorname{Sec}_{\sigma}^{*}(p)$, respectively.

Due to Theorem 9. Theorem 10 shall be established once we prove:

Theorem 13. As differential graded Lie algebras,

$$
\begin{gathered}
\left(\mathcal{H o m}\left(B^{\sharp}, L\right), D_{\phi},[,]\right) \cong\left(s^{-1} \mathcal{D e r}_{B}^{\phi}(B \otimes \Lambda V, B), \delta,[,]\right), \\
\left(\mathcal{H o m}\left(B_{+}^{\sharp}, L\right), D_{\phi},[,]\right) \cong\left(s^{-1} \mathcal{D e r}_{B}^{\phi}\left(B \otimes \Lambda V, B^{+}\right), \delta,[,]\right) .
\end{gathered}
$$

Proof. We only show the first assertion as the second has an identical proof. First note that, as graded vector spaces, $s^{-1} \operatorname{Der}_{B}^{\phi}(B \otimes \Lambda V, B) \cong \mathcal{H} o m\left(B^{\sharp}, L\right)$ :

$$
\begin{aligned}
& s^{-1} \operatorname{Der}_{B}^{\phi}(B \otimes \Lambda V, B)_{n}=\operatorname{Der}_{B}^{\phi}(B \otimes \Lambda V, B)_{n+1} \stackrel{\Theta}{\rightarrow}\left(V \otimes B^{\sharp}\right)_{n+1}^{\sharp} \\
& \stackrel{\nabla}{\leftarrow} \operatorname{Hom}_{n+1}\left(B^{\sharp}, s L\right)=\operatorname{Hom}_{n}\left(B^{\sharp}, L\right),
\end{aligned}
$$

in which $\nabla$ is the canonical isomorphism $\nabla \lambda(g \otimes \beta)=(-1)^{|g||\lambda|} g(\lambda(\beta))$. It is easily checked that this restricts to an isomorphism $s^{-1} \operatorname{Der}_{B}^{\phi}(B \otimes \Lambda V, B) \cong \mathcal{H o m}\left(B^{\sharp}, L\right)$. 
Next, we see that this is an isomorphism of Lie algebras. For that, choose $\varphi, \psi \in$ $\mathcal{D e r}_{B}^{\phi}(B \otimes \Lambda V, B)$ and $\lambda, \mu \in \mathcal{H o m}\left(B^{\sharp}, L\right)$ such that $\Theta \varphi=\nabla \lambda$ and $\Theta \psi=\nabla \mu$. Then, a straightforward computation shows that, for any $v \otimes \beta \in S, \Theta[\varphi, \psi](v \otimes \beta)=$ $\nabla s[\lambda, \mu](v \otimes \beta)$. It is worthwhile to mention that in this computation, the fact the following diagram commutes is essential:

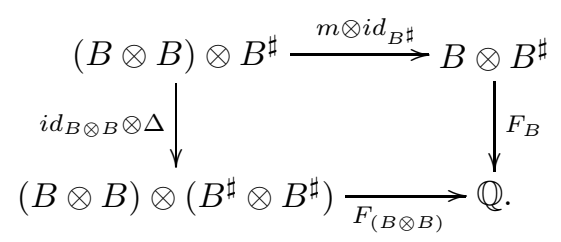

Here $m$ denotes multiplication and

$$
F_{B}(b \otimes \beta)=(-1)^{|b|} \beta(b), \quad F_{B \otimes B}\left(b \otimes b^{\prime} \otimes \beta \otimes \beta^{\prime}\right)=(-1)^{|b|\left|b^{\prime}\right|+|b|+\left|b^{\prime}\right|} \beta(b) \beta^{\prime}\left(b^{\prime}\right) .
$$

Finally, we prove that the isomorphism above commutes with differentials. According to Lemma 4, this is equivalent to the commutativity of the square

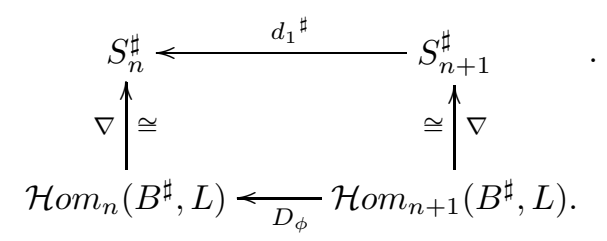

Let $\lambda \in \mathcal{H o m}_{n+1}\left(B^{\sharp}, L\right)$ and $\theta \in \mathcal{D e r}_{B}^{\phi}(B \otimes \Lambda V, B)_{n+1}$ such that $\nabla \lambda=\Theta \theta$. For any $v \otimes \beta \in S_{n}$, write $D v=d_{1} v+d_{2} v+\mathfrak{D} v+b$, where $\mathfrak{D} v=b^{\prime} w \in B^{+} \otimes V$. From now on, for simplicity in the notation and to make formulas clearer, we shall omit signs and write just \pm . However, a careful application of the Koszul convention leads to proper sign adjustments. On the one hand, a short computation shows that

$$
d_{1}^{\sharp}(\nabla \lambda)(v \otimes \beta)= \pm \beta\left(\theta\left(d_{1} v+d_{2} v+b^{\prime} w+b\right)\right) \pm\langle v, \lambda(\delta \beta)\rangle .
$$

On the other hand,

$$
\left(\nabla D_{\phi} \lambda\right)(v \otimes \beta)= \pm\left\langle v, \partial_{s L} \lambda(\beta) \pm \lambda(\delta \beta)-s[\tilde{\phi}, \lambda](\beta) \pm \mathcal{R}_{\lambda}(\beta)\right\rangle .
$$

Now, one easily sees that $\beta\left(\theta\left(d_{1} v\right)\right)= \pm\left\langle v, \partial_{s L} \lambda(\beta)\right\rangle$ while a direct computation, with the aid of formula (1) and the commutative square above, shows that $\beta(\theta(b))=0, \beta\left(\theta\left(d_{2}(v)\right)= \pm\langle v, s[\tilde{\phi}, \lambda](\beta)\rangle, \beta\left(\theta\left(b^{\prime} w\right)\right)= \pm\left\langle v, \mathcal{R}_{\lambda}(\beta)\right\rangle\right.$ and the theorem follows.

Next, we give an explicit and natural Lie model of the classical evaluation fibration.

Theorem 14. The fibration $\operatorname{Sec}_{\sigma}^{*}(p) \rightarrow \operatorname{Sec}_{\sigma}(p) \stackrel{e v}{\rightarrow} F$, in which $\operatorname{ev}(\gamma)=\gamma(*)$, is modeled by the exact sequence

$$
0 \rightarrow \mathcal{H o m}\left(B_{+}^{\sharp}, L\right) \stackrel{\operatorname{Hom}\left(i^{\sharp}, L\right)}{\longrightarrow} \mathcal{H o m}\left(B^{\sharp}, L\right) \stackrel{e v}{\longrightarrow} L \rightarrow 0
$$

in which $i$ : $B^{+} \hookrightarrow B$ and $e v(f)=f(1)$. Equivalently, it is modeled by

$$
0 \rightarrow \mathcal{L}_{*}^{\phi} \stackrel{j}{\longrightarrow} \mathcal{L}^{\phi} \stackrel{q}{\longrightarrow} L \rightarrow 0
$$


in which $j$ is the restriction of the inclusion $B^{+} \otimes L \hookrightarrow B \otimes L$ and $q$ the obvious projection.

Proof. Apply the functor $\operatorname{Der}_{B}^{\phi}(B \otimes \Lambda V,-)$ to the sequence $0 \rightarrow B^{+} \rightarrow B \rightarrow \mathbb{Q} \rightarrow 0$ to get an exact sequence

$0 \rightarrow s^{-1} \mathcal{D e r}_{B}^{\phi}\left(B \otimes \Lambda V, B^{+}\right) \rightarrow s^{-1} \operatorname{Der}_{B}^{\phi}(B \otimes \Lambda V, B) \rightarrow s^{-1} \operatorname{Der}_{B}^{\phi}(B \otimes \Lambda V, \mathbb{Q}) \rightarrow 0$.

By Theorem 9 (see also [4, Cor.7.2]), this is a Lie model of the evaluation fibration. Observe that here, $s^{-1} \mathcal{D e r}_{B}^{\phi}(B \otimes \Lambda V, \mathbb{Q}) \cong s^{-1}\left(V^{\sharp}\right)=s^{-1} s L=L$. To finish, apply Theorem 13 and the result follows.

\section{MAPPING SPACES}

The results proved in the earlier sections give, in particular, Lie models for pointed and free mapping spaces, when applied to the trivial fibration. In fact, let $X$ be a finite complex and let $f: X \rightarrow Y$ be a map modeled by the morphism $\phi: C^{*}(L)=(\Lambda V, d) \rightarrow B$ in which $L$ is a Lie model of $Y$ and $B$ a finite dimensional CDGA model of $X$. Hence $\phi$ is a retraction of the trivial KS-extension $B \rightarrow B \otimes \Lambda V$. In this particular context, Theorems 10, 14 and Corollary 12 read:

Corollary 15. The DGL's $\mathcal{H o m}\left(B^{\sharp}, L\right)$ and $\mathcal{H o m}\left(B_{+}^{\sharp}, L\right)$, or $\mathcal{L}^{\phi}$ and $\mathcal{L}_{*}^{\phi}$, are Lie models of $\operatorname{map}_{f}(X, Y)$ and $\operatorname{map}_{f}^{*}(X, Y)$ respectively. Moreover, the fibration $\operatorname{map}_{f}^{*}(X, Y) \rightarrow \operatorname{map}_{f}(X, Y) \stackrel{e v}{\rightarrow} Y$ is modeled by

$$
\begin{aligned}
& 0 \rightarrow \mathcal{H o m}\left(B_{+}^{\sharp}, L\right) \stackrel{\operatorname{Hom}\left(i^{\sharp}, L\right)}{\longrightarrow} \mathcal{H o m}\left(B^{\sharp}, L\right) \stackrel{e v}{\longrightarrow} L \rightarrow 0 \quad \text { or } \\
& 0 \rightarrow \mathcal{L}_{*}^{\phi} \stackrel{j}{\longrightarrow} \mathcal{L}^{\phi} \stackrel{q}{\longrightarrow} L \rightarrow 0 .
\end{aligned}
$$

Remark 16. Note that, for mapping spaces, the differential in these models simplifies to $D_{\phi}=D-a d_{\tilde{\phi}}$, i.e., $D_{\phi} f=\partial_{L} f+(-1)^{|f|} f \delta-[\tilde{\phi}, f]$. In fact, for the trivial KS-extension, $\mathcal{R}=0$ as $\mathfrak{D}$ vanishes.

Equivalently, in $\mathcal{L}^{\phi}$,

$$
\delta_{\phi}(a \otimes l)=d a \otimes l+(-1)^{|a|} a \otimes \delta l+\sum_{i}(-1)^{\left|b_{i}\right||l|} a b_{i} \otimes\left[l, l_{i}\right] .
$$

It is convenient to observe that, in the purely algebraic setting, for a given linear map of degree $-1, \varphi: B^{\sharp} \rightarrow L$ between a differential graded coalgebra and a DGL, $D_{\varphi}$ does not define a differential in $\operatorname{Hom}\left(B^{\sharp}, L\right)$. For that, a necessary and sufficient condition is that, with the usual differential $D$ and bracket [, ], the Maurer-Cartan equation is satisfied,

$$
D \varphi=\frac{1}{2}[\varphi, \varphi] .
$$

Moreover, those maps satisfying the Maurer-Cartan equation are exactly the twisting cochains.

With all of the above, we have a covariant algebraic construction to describe the rational homotopy type of mapping spaces and the corresponding evaluation fibrations. As a first strong application we see that the exponential law for spaces can be modeled by the corresponding exponential law in our context.

Let $B$ and $C$ be finite dimensional CDGA models of $X$ and $Y$ respectively, let $L$ be a DGL model of $Z$ and let $f: X \times Y \rightarrow Z$ be a map represented by 
$\phi: C^{*}(L)=\Lambda V \rightarrow B \otimes C$. Consider the linear maps of degree $-1, \tilde{\varphi}: C \rightarrow L$, $\tilde{\psi}: B \rightarrow \operatorname{Hom}\left(C^{\sharp}, L\right)$, defined by $\tilde{\varphi}(c)=\tilde{\phi}(1 \times c), \tilde{\psi}(b)(c)=\tilde{\phi}(b \otimes c)$. Then, we have:

Theorem 17. A Lie model for the exponential law

$$
\exp : \operatorname{map}_{f}(X \times Y, Z) \stackrel{\cong}{\rightrightarrows} \operatorname{map}_{f_{1}}\left(X, \operatorname{map}_{f_{2}}(Y, Z)\right)
$$

is the DGL isomorphism

$$
\Psi: \operatorname{Hom}^{\tilde{\phi}}\left(B^{\sharp} \otimes C^{\sharp}, L\right) \stackrel{\cong}{\rightarrow} \mathcal{H o m}^{\tilde{\psi}}\left(B^{\sharp}, \mathcal{H o m}^{\tilde{\varphi}}\left(C^{\sharp}, L\right)\right)
$$

induced by canonical adjunction.

Proof. Let $S, T, U$ be compactly generated spaces. Denote by $e v: \operatorname{map}(S, U) \times S \rightarrow$ $U$ the evaluation map and by $A:[T, \operatorname{map}(S, U)] \cong[T \times S, U]$ the bijection given by adjunction. Hence, two maps $h, k: T \rightarrow \operatorname{map}(S, U)$ are homotopic if and only if the composite maps $T \times S \stackrel{h \times i d}{\longrightarrow} \operatorname{map}(S, U) \times S \stackrel{e v}{\longrightarrow} U$ and $T \times S \stackrel{k \times i d}{\longrightarrow} \operatorname{map}(S, U) \times S \stackrel{e v}{\longrightarrow}$ $U$ are homotopic.

In particular, for $T=\operatorname{map}(X \times Y, Z), S=X$ and $U=\operatorname{map}(Y, Z)$, the exponential map exp: $\operatorname{map}(X \times Y, Z) \rightarrow \operatorname{map}((X, \operatorname{map}(Y, Z))$ is the only map up to homotopy making homotopy commutative the diagram

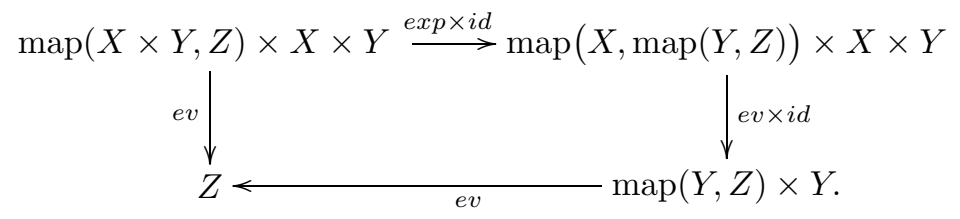

Now denote by $\left(\Lambda\left(V \otimes(C \otimes B)^{\#}\right), \tilde{d}\right)$ and $\left(\Lambda\left(\left(V \otimes C^{\#}\right) \otimes B^{\#}\right), \tilde{d}\right)$ the Haefliger models for $\operatorname{map}(X \times Y, Z)$ and $\operatorname{map}(X, \operatorname{map}(Y, Z))$. Recall also [3, 9, that a model for the evaluation map $e v: \operatorname{map}(Y, Z) \times Y \rightarrow Z$ is given by $\omega: \Lambda V \rightarrow \Lambda\left(V \otimes C^{\#}\right) \otimes C$, $\omega(v)=\sum_{i}\left(v \otimes \gamma_{i}\right) \otimes c_{i}$, with $\left\{c_{i}\right\}$ and $\left\{\gamma_{i}\right\}$ a basis of $C$ and its dual basis of $C^{\#}$ respectively. Denote by $\theta: C^{\#} \otimes B^{\#} \rightarrow(C \otimes B)^{\#}$ the linear isomorphism defined by $\theta(\gamma \otimes \beta)(c \otimes b)=(-1)^{|\beta||c|} \beta(b) \gamma(c)$. A simple computation shows that

$$
\Gamma=\Lambda(\theta \otimes 1):\left(\Lambda\left(\left(V \otimes C^{\#}\right) \otimes B^{\#}\right), \tilde{d}\right) \rightarrow\left(\Lambda\left(V \otimes(C \otimes B)^{\#}\right), \tilde{d}\right)
$$

is an isomorphism of differential graded algebras making commutative the diagram

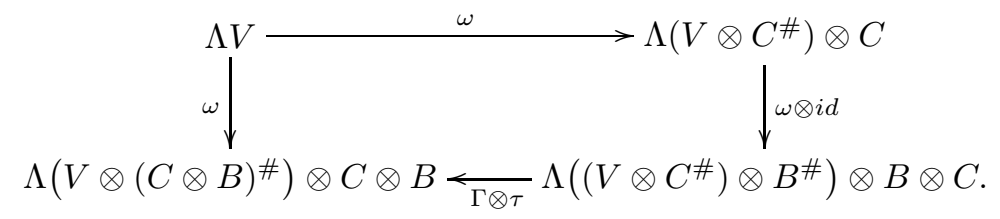

Here, $\tau(b \otimes c)=(-1)^{|b||c|} c \otimes b$. Since rationally, homotopy classes of maps between two spaces correspond in a bijective way to homotopy classes of maps between their models, this shows that $\Gamma$ is a Sullivan model for exp.

Now, using the isomorphism of Remark 11, $\Psi$ can be written as the canonical isomorphism $\Psi^{\prime}:(B \otimes C) \otimes L \rightarrow B \otimes(C \otimes L)$ defined by $\Psi^{\prime}((b \otimes c) \otimes \ell)=b \otimes(c \otimes \ell)$. The cochain algebra map $C^{*}\left(\Psi^{\prime}\right)$ is the above isomorphism $\Gamma$. Therefore $\Psi$ is a Lie model for exp. 
Another important application of our models is that rational homotopy groups of free and pointed mapping spaces together with their evaluation subgroups, i.e., (generalized) Gottlieb groups, can be effectively computed. We now present some results in this direction.

Proposition 18. For any 1-connected space $Y, \alpha \in \pi_{n}(Y), n \geq 1$, there is a natural isomorphism,

$$
\pi_{*} \operatorname{map}_{\alpha}\left(S^{n}, Y\right) \cong \operatorname{ker} a d_{\alpha} \oplus \operatorname{coker} a d_{\alpha}
$$

while $\pi_{*} \operatorname{map}_{\alpha}^{*}\left(S^{n}, Y\right) \cong \pi_{*}(Y)$.

More generally, if $X=\bigvee_{i \in I} S^{n_{i}}$ is a wedge of spheres and $\alpha_{i} \in \pi_{n_{i}}(Y), i \in I$, then

$$
\pi_{*} \operatorname{map}_{\left(\alpha_{i}\right)}(X, Y) \cong\left(\bigcap_{i} \operatorname{ker} a d_{\alpha_{i}}\right) \oplus\left(\bigoplus_{i} \operatorname{coker} a d_{\alpha_{i}}\right)
$$

and $\pi_{*} \operatorname{map}_{\left(\alpha_{i}\right)}^{*}(X, Y) \cong \bigoplus_{i} \pi_{*}(Y)$.

Here, $a d_{\alpha}: \pi_{*}(Y) \rightarrow \pi_{*}(Y)$ denotes the adjoint of the Whitehead bracket, $a d_{\alpha}(\beta)$ $=[\alpha, \beta]$.

Proof. Fix $L$ a Lie model of $Y$ and consider the CDGA model of $S^{n}, B=\left(\Lambda a /\left\langle a^{2}\right\rangle\right.$, $0),|a|=n$. Next, choose $\tilde{\phi}: B^{\sharp}=\mathbb{Q} \oplus\langle\beta\rangle \rightarrow L$ of the form $\tilde{\phi}(\beta)=x$ in which $x \in L_{n-1}$ is a cycle representing $\alpha \in \pi_{n-1}\left(\Omega S^{n}\right)=\pi_{n}\left(S^{n}\right)$. Hence, if we denote by $e L$ the suspension $s^{n} L, B \otimes L \cong L \oplus e L$ and as such,

$$
\delta_{\phi}(y, z)=\left(\delta y,(-1)^{|a|} \delta z+(-1)^{n|y|}[y, x]\right) .
$$

Hence the map $\Psi: \pi_{*}\left(\Omega \operatorname{map}_{\alpha}\left(S^{n}, Y\right)\right)=H_{*}(B \otimes L) \rightarrow$ ker $a d_{\alpha}$ defined by $\Psi[(y, z)]$ $=[y]$ is obviously surjective and $\operatorname{ker} \Psi \stackrel{\cong}{\rightrightarrows} \operatorname{coker} a d_{\alpha}$ via $[(y, z)] \mapsto \overline{[z-[x, t]]}$ with $\delta t=y$. In the same way, consider the CDGA model of $X=\bigvee_{i \in I} S^{n_{i}}, B=$ $\left(\Lambda\left(b_{i}\right) /\left(b_{j} b_{k}\right), 0\right),\left|b_{i}\right|=n_{i}, j, k \in I$. Let $\left\{\beta_{i}\right\}$ be a basis of $B^{\sharp}$ and choose $\tilde{\phi}\left(\beta_{i}\right)=x_{i}$, a cycle in $L_{n_{i}-1}$ representing $\alpha_{i}$. In $B \otimes L \cong L \oplus\left(\bigoplus_{i} e_{i} L\right)$,

$$
\delta_{\phi}\left(y_{0}, \sum_{i} y_{i}\right)=\left(\delta y_{0}, \sum_{i}(-1)^{n_{i}} \delta y_{i}+(-1)^{n_{i}\left|y_{0}\right|}\left[y_{0}, x_{i}\right]\right) .
$$

A similar argument to the previous case proves the desired general result.

Remark 19. Observe that, for $X=\bigvee_{i \in I} S^{n_{i}}$, the model of the evaluation fibration $\pi_{*} \operatorname{map}_{\left(\alpha_{i}\right)}^{*}(X, Y) \rightarrow \pi_{*} \operatorname{map}_{\left(\alpha_{i}\right)}(X, Y) \rightarrow Y$ is then $\bigoplus_{i} e_{i} L \rightarrow L \oplus\left(\bigoplus_{i} e_{i} L\right) \rightarrow L$, and the evaluation subgroup of $\pi_{*}(Y)$ is $\bigcap_{i} \operatorname{ker} a d_{\alpha_{i}}$. This is coherent with the topology because the class of a map $g: S^{n} \rightarrow Y$ is in the image of the evaluation if and only if $g \vee f: S^{n} \vee X \rightarrow Y$ extends to the product, which means that $\left[g, x_{i}\right]=0$ for all $i$. Note also that the connecting map in the rational long exact homotopy sequence is given by

$$
\partial: H_{*}(L) \rightarrow \bigoplus_{i} H_{*}\left(e_{i} L\right), \quad \partial \gamma=\sum_{i}(-1)^{n_{i}|\gamma|+1}\left[\gamma,\left[x_{i}\right]\right] .
$$

The previous examples give the flavor of the general situation. Suppose $B$ is 1-connected and filter the model $B \otimes L$ of the mapping space given in Remark 11 by the differential ideals $F_{p}=B^{\geq p} \otimes L$. This filtration induces a spectral sequence of graded Lie algebras in which $E_{p}^{1}=B^{p} \otimes H_{*}(L)$ and $E_{p}^{2}=H^{p}(B) \otimes H_{*}(L)$. If $L=(L, 0)$, the differential of any stage is easy to compute: If $\left\{h_{j}\right\}$ are cocycles 
in $B$ such that the classes $\left\{\left[h_{j}\right]\right\}$ form a basis of $H^{*}(B)$ and $\tilde{\phi}\left(h_{j}\right)=\ell_{j}$, then $d^{2}([a] \otimes \ell)=\sum_{j}\left[a h_{j}\right] \otimes\left[\ell, \ell_{j}\right]$, and higher order differentials have a similar form.

From this we see that, in this context, the rate of growth of the rank of $\pi_{*} \operatorname{map}_{f}(X, Y)$ is the same as that of $\pi_{*}(Y)$. Recall that the log index of a graded vector space $V$ is defined as the limit

$$
\log \operatorname{index} V=\limsup _{k} \frac{\log \operatorname{dim} V_{k}}{k} .
$$

Recall also that a simply connected space $Y$ is coformal if its rational homotopy type depends only on its rational homotopy Lie algebra. In other words, if its minimal model is isomorphic to the cochain algebra on the graded Lie algebra $\pi_{*}(\Omega Y) \otimes \mathbb{Q}$.

Then, assuming $X$ and $Y$ are simply connected, we deduce:

Theorem 20. Let $Y$ be a coformal space of finite Lusternik-Schnirelmann category. Then:

(i) If $\operatorname{Im} \tilde{\phi} \subset I$ with $\log$ index $I<\log$ index $L$, then

$$
\log \operatorname{index} \pi_{*}\left(\operatorname{map}_{f}(X, Y)\right) \otimes \mathbb{Q}=\log \operatorname{index} \pi_{*}(Y) \otimes \mathbb{Q} .
$$

(ii) If the Lie algebra $\pi_{*}(\Omega Y) \otimes \mathbb{Q}$ has an infinite number of generators, then $\pi_{*}\left(\operatorname{map}_{f}(X, Y)\right) \otimes \mathbb{Q}$ is also infinite dimensional.

Proof. Recall that for any simply connected space $Y$ of finite Lusternik-Schnirelmann category $m$ there are at most $m$ numbers that can appear as log index $I$ for some ideal $I$ in $L=\pi_{*}(\Omega Y) \otimes \mathbb{Q}$ [8]. Moreover, the sum of the ideals $I_{j}$ with $\log$ index $I_{j}<b=\log$ index $L$ is an ideal with $\log$ index $<b$ [8]. Since $Y$ is coformal, a Lie model for $Y$ is $(L, 0)$.

Next, observe that, in the spectral sequence defined above, the image of $d^{2}$ is contained in the ideal generated by $\operatorname{Im} \tilde{\phi}$ and thus, in $I$. To describe $d^{3}$, let $\sum_{k}\left[a_{k}\right] \otimes u_{k}$ be a $d^{2}$-cocycle, i.e., $\sum_{j, k}\left[a_{k} h_{j}\right] \otimes\left[u_{k}, \ell_{j}\right]=0$. Then, there are elements $c_{j, k} \in B$ such that

$$
\sum_{j, k} a_{k} h_{j} \otimes\left[u_{k}, \ell_{j}\right]=d\left(\sum_{j, k} c_{j, k} \otimes\left[u_{k}, \ell_{j}\right]\right) .
$$

Then,

$$
d^{3}\left(\sum_{k}\left[a_{k}\right] \otimes u_{k}\right)=\sum_{j, k, s}\left[c_{j, k} h_{s}\right] \otimes\left[\left[u_{k}, \ell_{j}\right], \ell_{s}\right],
$$

which is also contained in the ideal generated by $\operatorname{Im} \tilde{\phi}$ and thus, in $I$. As this fact occurs in general at each stage, the log index of the image of any differential $d^{r}$ is, by hypothesis, strictly less than the log index of the complex. Therefore $\log$ index $E^{r}=\log$ index $L$ for each $r$. Since the spectral sequence has only a finite number of terms, (i) follows.

For (ii) choose a nonzero cohomology class of maximal degree $\alpha \in H^{*}(B)$ and an indecomposable class $\gamma \in L$ with $|\gamma|>|\alpha|+1$. Then, $\alpha \otimes \gamma$ is an element of $E^{2}$-term of the spectral sequence which survives at any stage as $\gamma$ is indecomposable. The result is obtained by iterating the process with a sequence $\gamma_{i} \in H_{*}(L)$ of different degrees.

Observe that the condition $\operatorname{Im} \tilde{\phi} \subset I$ can be weakened, assuming only that a set of cycles representing $H^{*}(B)$ is sent to $I$ via $\tilde{\phi}$. 


\section{ACKNOWLEDGMENT}

We thank the referee for his/her suggestions and remarks.

\section{REFERENCES}

[1] A. K. Bousfield and V. K. A. M. Gugenheim, On PL de Rham theory and rational homotopy type, Mem. Amer. Math. Soc., 179 (1976). MR0425956 (54:13906)

[2] E. H. Brown and R. H. Szczarba, On the rational homotopy type of function spaces, Trans. Amer. Math. Soc., 349 (1997), 4931-4951. MR1407482 (98c:55015)

[3] U. Buijs and A. Murillo, Basic constructions in rational homotopy theory of function spaces, Annales de l'Inst. Fourier, 56(3) (2006), 815-838. MR2244231 (2007h:55009)

[4] U. Buijs and A. Murillo, The rational homotopy Lie algebra of function spaces, to appear in Comment. Math. Helv.

[5] F. E. A. da Silveira, Rational homotopy theory of fibrations, Pacific Journal of Math., 113(1) (1984), 1-34. MR745592 (86c:55013)

[6] Y. Félix, Rational category of the space of sections of a nilpotent bundle, Comment. Math. Helvetici, 65 (1990), 615-622. MR1078101 (91j:55012)

[7] Y. Félix, S. Halperin and J.C. Thomas, Rational Homotopy Theory, Graduate Texts in Math. 205, Springer, 2001. MR 1802847 (2002d:55014)

[8] Y. Félix, S. Halperin and J.C. Thomas, Structure of homotopy Lie algebras, Accepted for publication in Comment. Math. Helv.

[9] A. Haefliger, Rational homotopy of the space of sections of a nilpotent bundle, Trans. Amer. Math. Soc., 273 (1982), 609-620. MR667163 (84a:55010)

[10] P. Hilton, G. Mislin and J. Roitberg, Localization of nilpotent groups and spaces, North Holland Mathematics Studies 15, North Holland, 1975. MR0478146 (57:17635)

[11] G. Lupton and S. Smith, Rational evaluation subgroups of a map II : Quillen models and adjoint maps, Journal of Pure and Applied Algebra, 209 (2007), 173-188. MR 2292125 (2008c:55018)

[12] J. M. Möller, Nilpotent spaces of sections, Trans. Amer. Math. Soc., 303 (1987), 733-741. MR902794 (88j:55007)

[13] H. Scherer and D. Tanré, Homotopie modérée et tempérée avec les coalgèbres. Applications aux espaces fonctionnels, Arch. Math., 59(2) (1992), 130-145. MR1170636 (93k:55016)

[14] D. Sullivan, Infinitesimal computations in topology, Publ. Math. de l'I.H.E.S., 47 (1978), 269-331. MR0646078 (58:31119)

[15] R. Thom, L'homologie des espaces fonctionnels, Colloque de topologie algébrique, Louvain, (1957), 299-39.

[16] M. Vigué-Poirrier, Sur l'homotopie rationnelle des espaces fonctionnels, Manuscripta Math., 56 (1986), 177-191. MR850369 (87h:55009)

Departamento de Matemática Aplicada, Campus El Ejido, Universidad de Málaga, 29000 MÁlaga, SPAin

E-mail address: urtzi@agt.cie.uma.es

Institut de Mathématique Pure et Appliquée, Chemin du Cyclotron, 2, Université Catholique de Louvain, B-1348 Louvain-la-Neuve, Belgique

E-mail address: felix@math.ucl.ac.be

Departamento de Álgebra, Geometría y Topología, Universidad de Málaga, Ap. 59, 29080 Málaga, Spain

E-mail address: aniceto@agt.cie.uma.es 